\title{
EVALUATION OF SYSTEMATIC ERRORS IN THE AVALANCHE LOCALIZATION ALONG THE WIRE WITH CATHODE STRIPS READ-OUT MWPC
}

\author{
F. PIUZ, R. ROOSEN * \\ CERN, Geneva, Switzerland
}

and

\section{J. TIMMERMANS}

NIKHEF-H, Amsterdam, The Netherlands

Received 14 October 1981

Systematic errors on the avalanche localization along a wire have been investigated and measured in a centroid chamber array described here. The use of discrete sampling by cathode strips may result in a systematic error which can be of the order of the chamber resolution. It is shown both by calculation and experiment that this error can be minimized by an appropriate choice of a "bias level" in the algorithm used for coordinate calculation. Another source of systematic error is also discussed.

\section{Introduction}

We describe the construction and performance of a beam telescope consisting of 6 cathode strip read-out proportional chambers as to provide 3 sets of $(y, z)$ coordinates of an incident track, the avalanche being localized along the wire. Various sources of errors in the coordinate determination are investigated: measurement errors as well as those resulting from the appliation of the Centre Of Gravity (COG) method. They will be referred to later on, as, respectively, "external and "internal" errors. Special attention has been paid to their evaluation as a function of the coordinate along the wire. The result of this investigation is that appropriate corrections can be found allowing an approximately uniform accuracy to be achieved across the entire chamber surface. At the same time, it leads to a practical method of optimization of the construction and operation parameters of such a device.

The telescope has so far been used in two different experimental set-ups. In the first they served to define the trajectory of the particles in a test beam at the CERN Proton Synchrotron. This set-up was used to check and calibrate high precision drift chambers which are developed for an experiment to measure the elastic scattering cross section at the CERN pp Collider [1]. Results of the accuracies obtained in these drift chambers will be published elsewhere [2].

\footnotetext{
* Now at Brussels University, Brussels, Belgium
}

The second use was the installation in a hybrid experiment (NA19), designed to search for the direct production of beauty particles by a $320 \mathrm{GeV} / c$ incident beam of negatively charged pions on an emulsion stack [3]. The telescope was needed to measure accurately the location of the incident track with respect to the stack. In order to enhance the signal, the chambers were triggered whenever the downstream spectrometer detected two or more muons. To obtain the required sensitivity, the telescope together with the emulsion stack was mounted on a platform which could be moved across the beam $\left(10 \times 50 \mathrm{~mm}^{2}\right)$ so as to irradiate the emulsion uniformly with a track density of $2000 / \mathrm{mm}^{2}$. Under these circumstances it is obvious that the emulsion scanning effort is closely related to the precision with which the coordinate of the incident particle is measured at the entrance of the stack.

In both applications one has to match, using single tracks, the prediction given by the centroid system with the measurement of an external device, i.e. the drift chamber or the emulsion stack, the latter having a resolution of a few micrometers. It is therefore very important to know and control the accuracy of the centroid system with respect to an external reference frame.

The COG determination by itself has an excellent intrinsic resolution (of the order of a few tens of microns), so that it is very difficult to use any other known type of detector to serve as an external ereference. We therefore present a method involving the use of identical centroid chambers only. Its principle is to measure 
accurately known displacements of one chamber with respect to the two others; this will also allow the easy detection of systematic errors in the COG determination.

\section{Evaluation of the centre of gravity of the induced charge}

Charpak and coworkers have demonstrated that accuracies as good as $50 \mu \mathrm{m}$ r.m.s. can be obtained in the direction along the anode wire for trajectories of minimum ionizing particles orthogonal to the detection plane having a cathode subdivided into strips [4,5]. In a theoretical approach, Gatti et al. [6] have developed an optimization of the chamber geometry parameters: taking into account the series white noise of the input amplifiers associated with each cathode strip, they have shown that a minimum variance is achievable, depending on the parameter ratios $2 a / D$ and $R / D$, where $2 a$ is the cathode strip pitch, $D$ the distance between the wire and cathode planes and $R$ the wire radius. The value of the minimum is found to be dependent on the position of the avalanche with respect to the cathode strip array.

In addition to the stochastic errors one has to consider for a given chamber geometry the systematic errors, associated with the choice of the algorithm used for the calculation of the coordinate along the wire. An obvious choice is

$\bar{z}=\sum_{i} A_{i} z_{i} / \sum_{i} A_{i}$,

where the $A_{i}$ 's are the pulse heights measured on a sequence of strips at positions $z_{i}=i \cdot 2 a$. The use of such a discrete set of measurements to find the COG of a continuous distribution is well known for introducing a systematic error which depends on the relative position of the physical impact point on this discrete array. This has been demonstrated for example in the localization of electromagnetic showers using a strip or cell segmented calorimeter [7]. In the case of track localization by the COG determination in a proportional chamber, Radeka et al. [8] measured such a differential non-linearity by means of a scan with a point source: they observed a sizeable periodic effect for $2 a / D=1.1$, while for $2 a / D=0.76$ stochastic error sources were dominant.

Endo et al. [9] calculated, under certain model assumptions, the shape of the induced charge distribution (sect. 3) and from this the systematic error in the determination of the charge centre for six different estimation algorithms. The errors thus calculated were used to correct their measurements. As the corrections to be applied were rather large and also depend heavily on the exact knowledge of the induced charge distribution, we preferred to search for an algorithm which leads to small systematic errors.

As a starting point, we used a modification of relation (1), already proposed by Charpak et al. [4]. The COG of a cluster of adjacent induced charges is written as

$$
\begin{aligned}
\bar{z}=\sum_{i}\left(A_{i}-B\right) z_{i} / \sum_{i}\left(A_{i}-B\right) & \\
& \text { for }\left(A_{i}-B\right)>0
\end{aligned}
$$

where $B$ is a suitably chosen bias level, introduced to reduce the effect of pick-up and electronics noise. In this reference best results were obtained with a bias level proportional to the total charge induced $B=b \Sigma A_{i}$. for a value of $b$ between 0.005 and 0.025 .

Our calculations are based on the analytical shape of the planar distribution of the induced charge given by Gatti et al. [6]. The formula includes the chamber geometry parameters, optimized or not, such as $D, 2 a$. $R$ and $s$ (the wire pitch). They were taken to be the ones of our chambers (sect. 4). An experimental verification of the calculated pulse shape is given in sect. 3. The systematic errors in the COG determination were calculated using relation (2). the results are shown in fig. 1 as a function of the position of the charge centre and for various values of the bias level $b$. In general, the error is found to be largest, but of opposite sign, when the charge centre is located at $1 / 4$ or $3 / 4$ of the strip width $2 a$, and to be zero (independent of the bias level) when located at the centre of a strip or just between two strips. The periodicity is thus one strip width. In fig. 2 we show the systematic error as a function of the bias $b$ for a charge centre located at $1 / 4$ of the strip width. At all positions across the strip one observes this same behaviour, i.e. three values of $b$ for which the systematic error is zero. However, only the lowest value of the three $(b=0.015)$ is approximately the same for all charge centre positions. We note that for $b=0.025$, the value used in ref. 4 , the systematic error can reach a maximum value of $0.006 \times$ stripwidth, i.e. $30 \mu \mathrm{m}^{*}$. comparable to their intrinsic resolution. For our geometry, this figure drops to only $16 \mu \mathrm{m}$ for a bias level of 0.015 .

Finally, we show in fig. 3 the influence of the parameter $2 a / D$ on the size of the systematic error at the $1 / 4$ position and for zero bias. One can see that this parameter has a drastic effect on the systematic error for values $2 a / D>1.1$; for values $2 a / D$ slightly smaller than 1 , one obtains very small systematic errors, in agreement with the measurements of Radeka and Boie [8]. The optimization by Gatti et al. [6] suggests $2 a / D$ $\simeq 1$.

* For a wire spacing of $2 \mathrm{~mm}$, ths error may become as big as $65 \mu \mathrm{m}$ for $b=0.025$. 


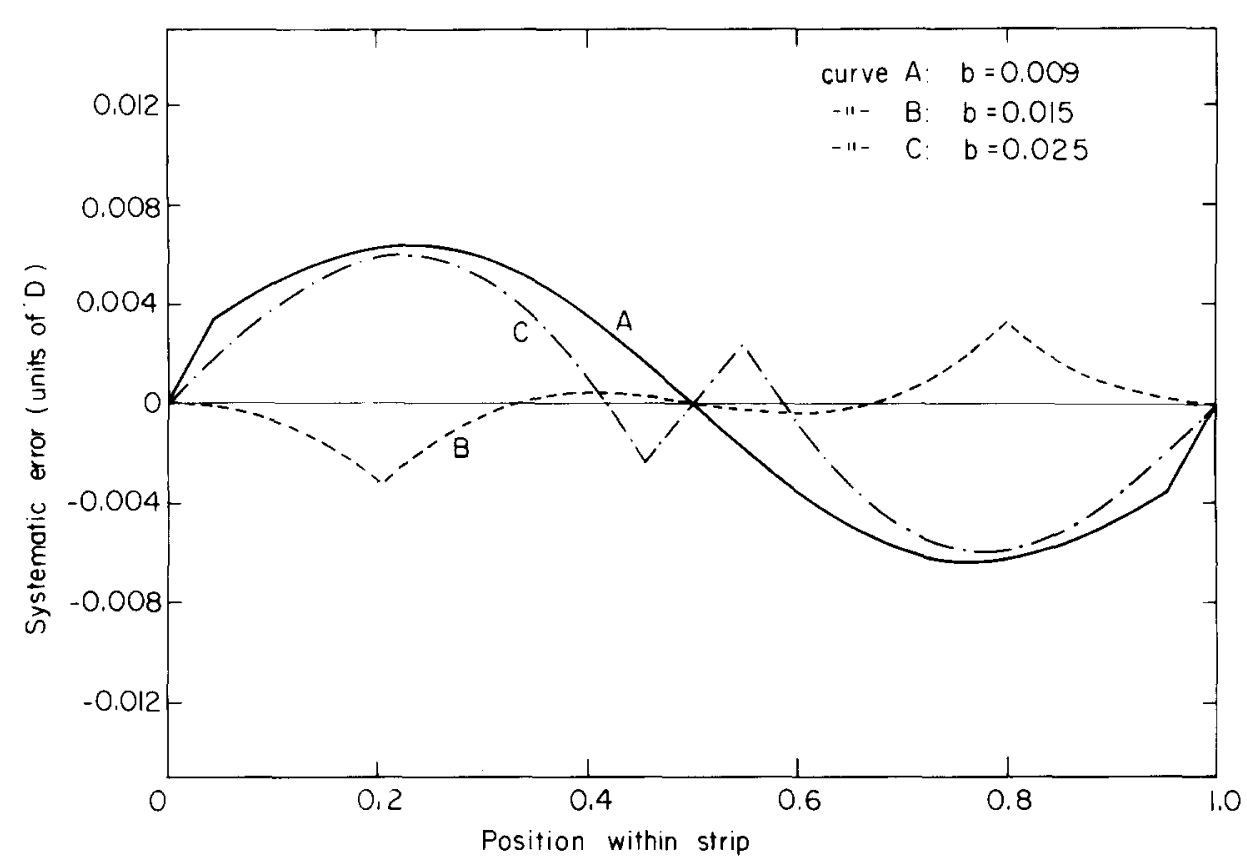

Fig. 1. Systematic error across a strip for various bia levels.

So far, we have presented a simple evaluation of the systematic errors in the COG determination using algorithm (2). With the particular value of the bias level $b=0.015$, the algorithm is adequate for our needs since the systematic errors are small compared to the expected chamber resolution. In the next section we will present an experimental support to these calculations.

\section{Experimental results}

\subsection{Method of measurement}

The experimental set-up (fig. 4) for the measurement of the resolution in the determination of the charge centroid consisted of a set of three identical chambers.

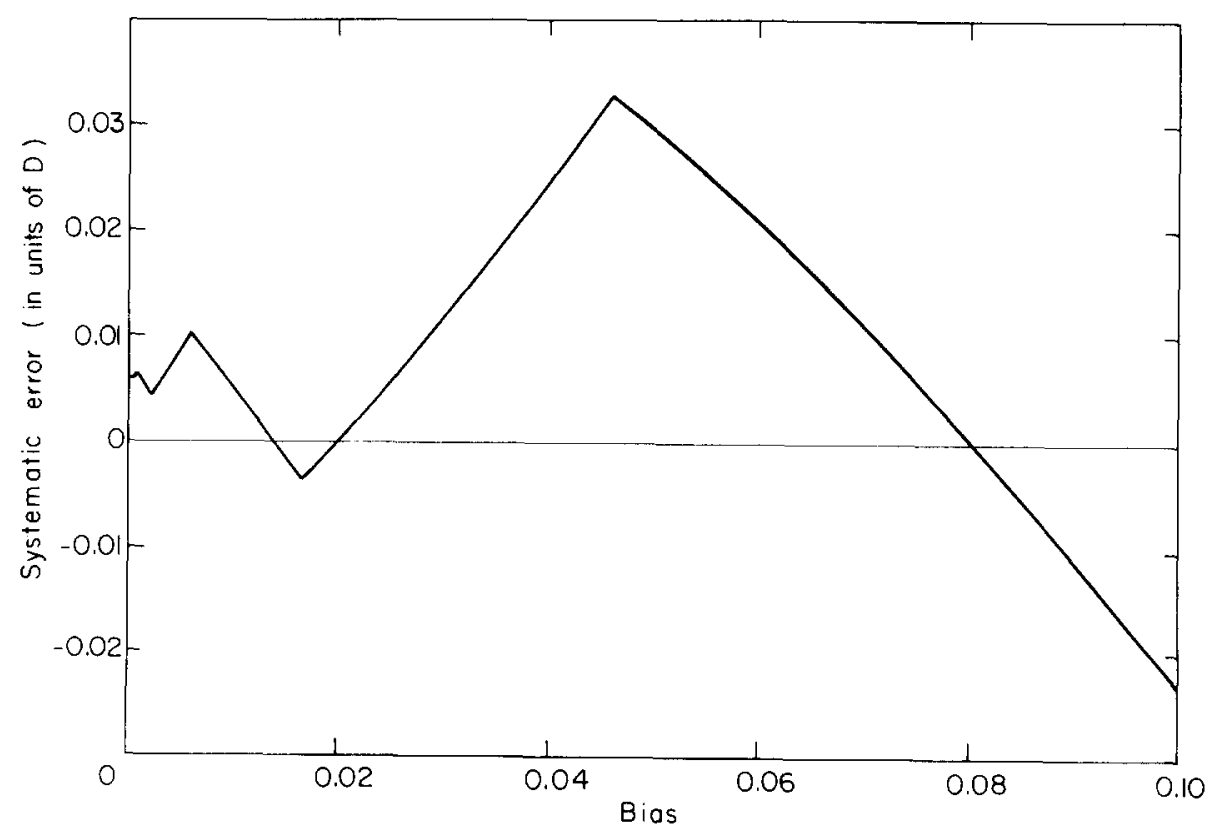

Fig. 2. Systematic error as a function of the bias for a charge centre located at $1 / 4$ of the strip width. 


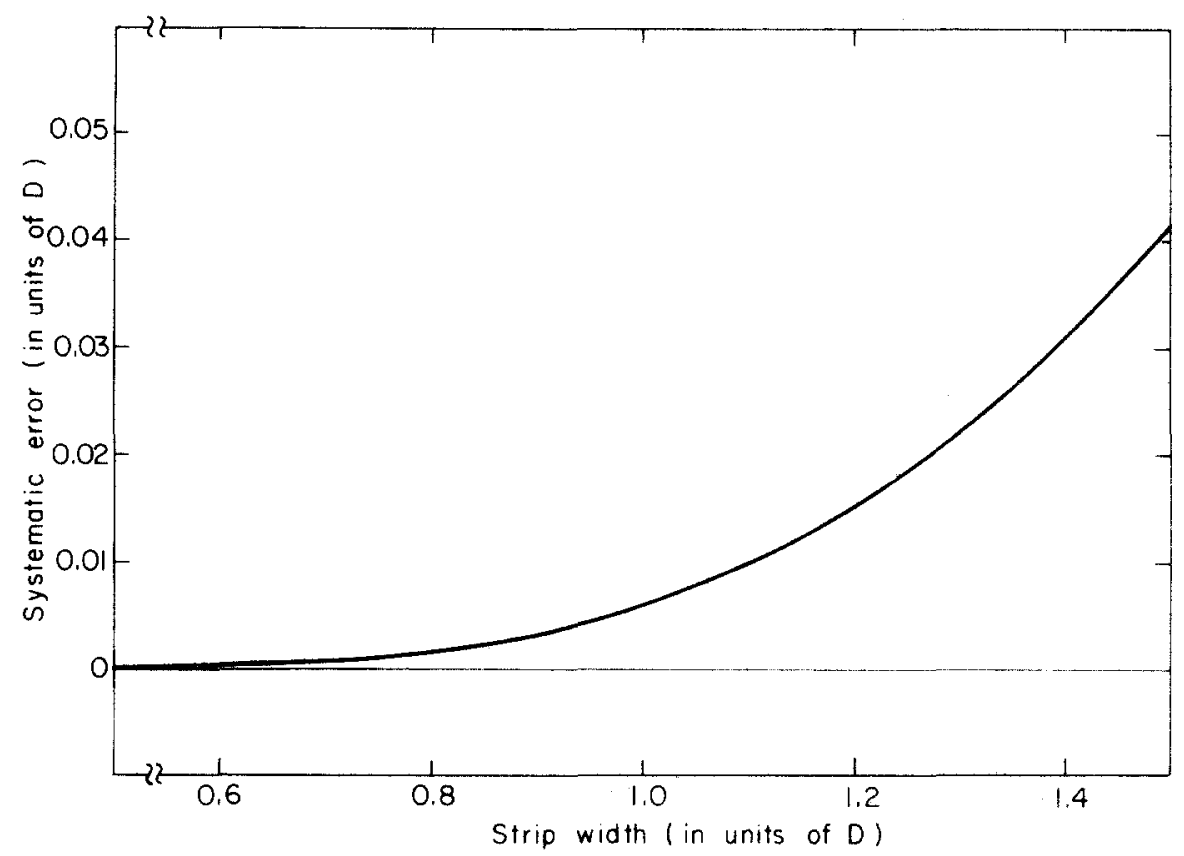

Fig. 3. Systematic error as a function of the strip width/gap width ratio, calculated for a charge centre located at $1 / 4$ of the strip width for zero bias.

Detailed features on their construction and operation are found in sect. 4. They were installed in a $10 \mathrm{GeV} / c$ $\pi^{-}$beam at the CERN Proton Synchrotron, oriented such that the vertical coordinates were measured (horizontal strips and vertical wires). The beam was orthogonal to the wire plane, with a divergence of $\sim 2 \mathrm{mrad}$ fwhm and an area of $\sim 4 \mathrm{~cm}^{2}$. The two outer chambers $C_{1}$ and $C_{3}$ were fixed to an external reference frame while the central one $C_{2}$ was movable along the vertical direction with a precision of $\pm 10 \mu \mathrm{m}$ with respect to $C_{1}$ and $\mathrm{C}_{3}$. The distance between anode wire planes was $110 \mathrm{~mm}$.

The resolution of the chamber is studied by looking at the distribution of the residuals $R_{z}$ of the central chamber. This quantity is defined as the difference between the measured coordinate $z_{2}$ and the predicted

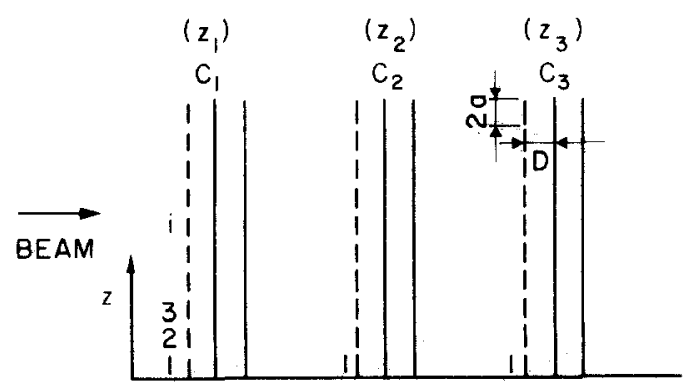

Fig. 4. Experimental set-up (side view, $\Delta z_{2}=0$ ). coordinate obtained by linear interpolation between the measured coordinates $z_{1}$ and $z_{3}$. Each of these measurements might be affected either by constant quantities $l_{1}$, $l_{2}$ and $l_{3}$ which result from slight vertical shifts and rotations in the chamber alignment, or (and) by a known displacement $\Delta z_{2}$ of the ehamber $C_{2}$. Then, one has

$R_{z}=\frac{z_{1}+z_{3}}{2}-z_{2}+\frac{l_{1}+l_{3}}{2}-l_{2}+\Delta z_{2}$.

The position of the centre of the $R_{z}$ distribution provides a measurement of the combination of the shift terms and of $\Delta z_{2}$. Assuming that the three measurements are not correlated and have equal error distributions per plane with a standard deviation $\sigma$ (r.m.s.), the full width half-maximum (fwhm) of the residual distribution will be equal to $2.36 \sigma \sqrt{(3 / 2)}$ as seen from relation (3).

\subsection{Results}

The following results on chamber performance have been obtained at a beam intensity of $\sim 1 \times 10^{5}$ particle/s - strip.

In fig. 5 , is shown the $R_{z}$ distribution as a function of the measured coordinate $z_{2}$ with the central chamber $C_{2}$ in a position such that the cathode strips boundaries are well aligned in the three chambers. In this case, $\Delta z_{2}=0$, all the possible effects of systematic errors are masked as they will have the same amplitude and sign in the 


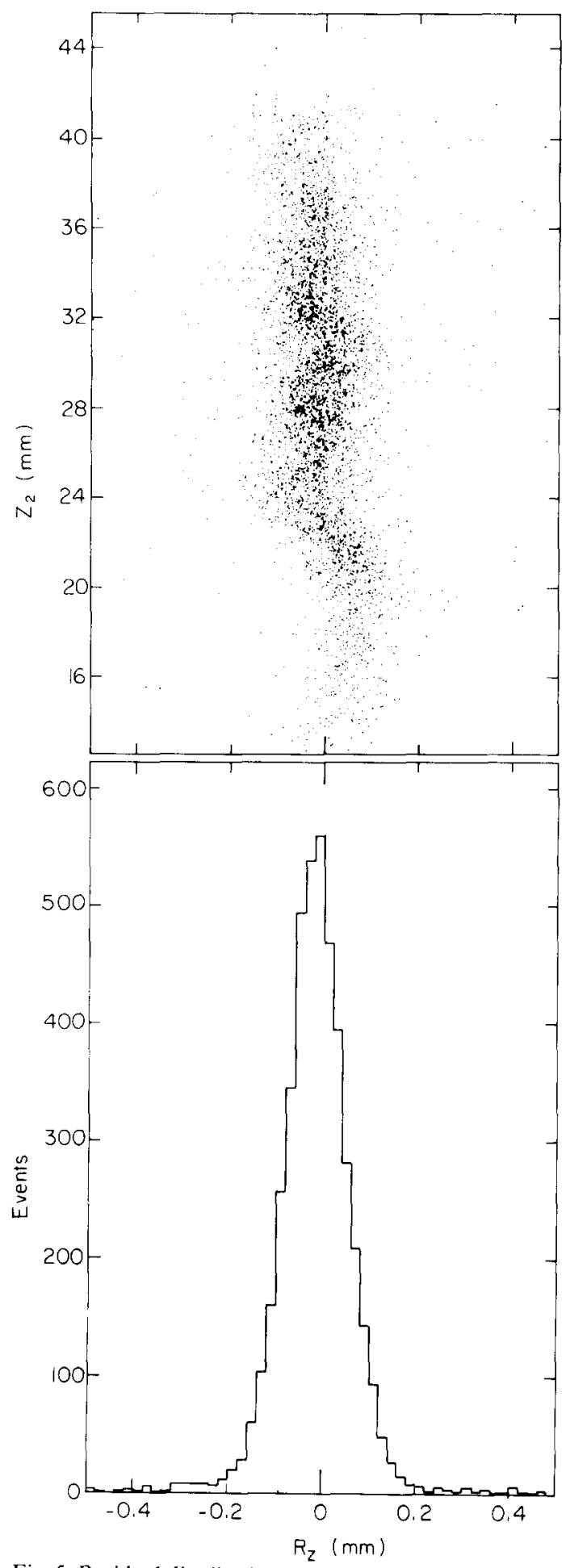

Fig. 5. Residual distribution vs the measured coordinate and its projection. All three chambers are aligned.

three chambers for particle trajectories perpendicular to the chambers at any $z$. This situation represents of course only one of the numerous choices of geometry but is nevertheless frequently chosen for practical pur- poses. This resulting $R_{z}$ distribution has a fwhm of 150 $\mu \mathrm{m}$, that is a standard deviation of $52 \mu \mathrm{m}$ per plane. It is centred around zero for all $z$ coordinates, indicating that there are no large systematic errors due to one or several but non correlated defective channels. The bias level used for the COG determination was $b=0.025$. Similar results were obtained for bias values between 0.005 and 0.025 . This accuracy is comparible with the one obtained by Charpak et al. [4], using a different signal processing.

\subsubsection{Example of a masked "external" systematic error}

As an illustration, we will demonstrate the effect of such a usual external source, an electronic cross talk. In fact, it was measured at a level of $1.4 \%$, between the two channels of each pair of preamplifiers, while a negligible contribution was found between any two channels not within a grouped pair. This configuration was quite homogeneous through the whole chamber. This asymmetric effect will appear fully correlated and at its maximum amplitude when the central chamber is displaced by one cathode strip width (equivalent to one electronic channel) with respect to $C_{1}$ and $C_{3}$, i.e. in our geometry $\Delta z_{2}=5 \mathrm{~mm}$. The corresponding $R_{z}$ distribution, function of $z_{2}$, is shown in fig. $6 \mathrm{a}$. The observed ondulation is due to the fact that the systematic error on the predicted and measured coordinates are now approximately equal in size but opposite in sign. A simple correction to the measured pulse heights of the form

$$
\begin{aligned}
& A_{i+1, \text { true }}=A_{i=1, \text { meas }}-0.013 A_{i, \text { meas }}, \\
& A_{i, \text { true }}=A_{i, \text { meas }}+0.015 A_{i+1, \text { meas }}
\end{aligned}
$$

(for $i$ odd),

is sufficient to eliminate to a large extent this systematic error (fig. $6 \mathrm{~b}$ ): the resulting resolution is $\sigma=62 \mu \mathrm{m}$ per plane. All the following results are obtained from measurements corrected in this way.

\subsubsection{Measurements of the "internal" systematic errors (COG determiation)}

Finally, we report on the verifications of the calculations of sect. 2. We start by presenting an experimental check of the formula given by Gatti et al. [6] which describes the shape of the induced charge distribution of the cathode. For each reconstructed track of coordinate $z$, we plot in fig. 7a the amplitudes of the hit strips as a function of the distance between the centre of the strip and the measured centre of gravity. An "average" value can be drawn (fig. 7b) by taking the most probable value of the Landau shaped distribution obtained by the ordinate projections made at different intervals of the abscissa. The result is then compared with the charge distribution functions calculated by Gatti et al. [6] (curve A), Endo et al. [9] (curve B) who neglected the effect of anode wires, Lee et al. [10] (curve C), who assumed a continuous anode plane and Fischer et al. [11] (curve D) who neglected the effects of both anode 


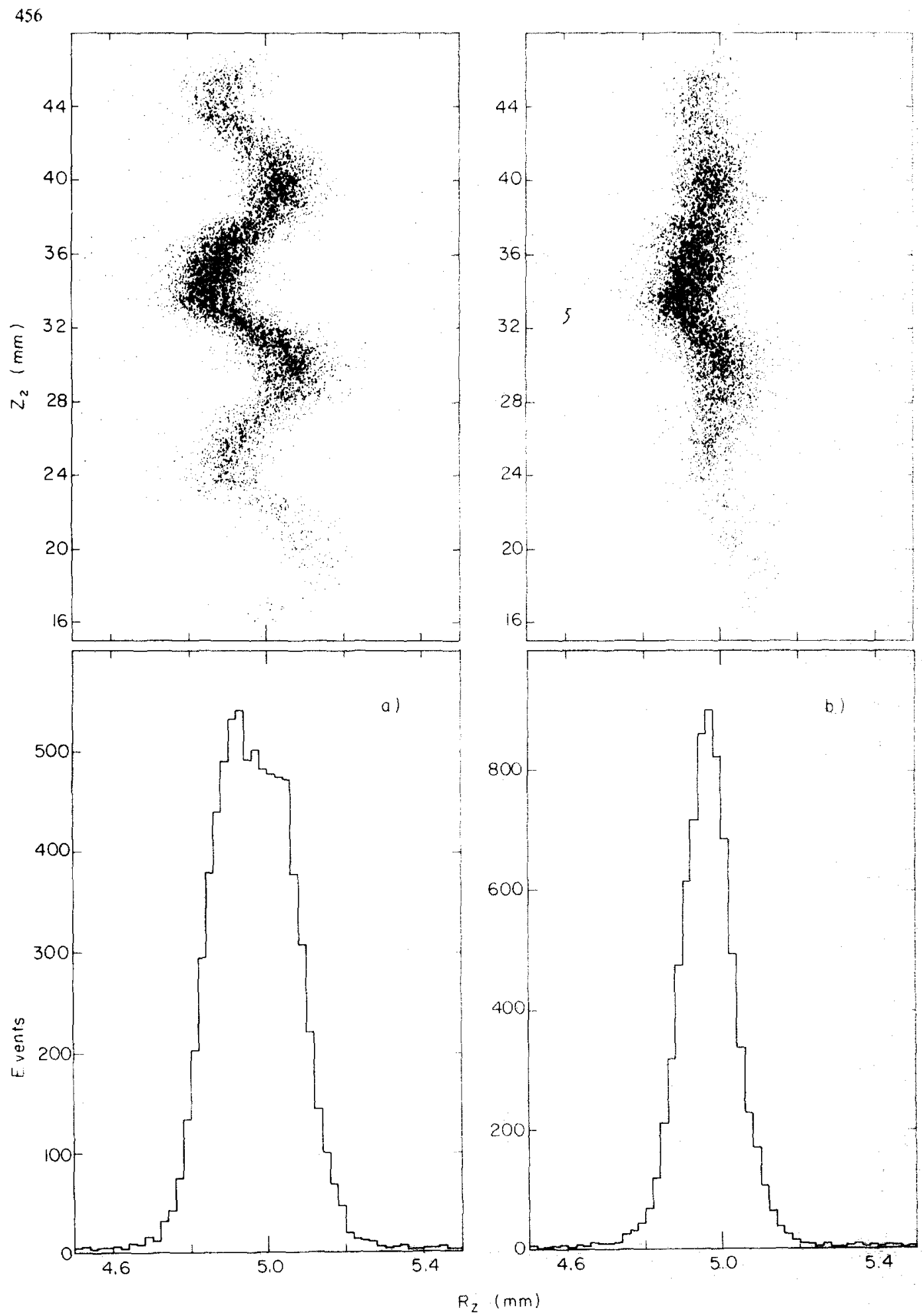

Fig. 6. Residual distribution vs the measured coordinate in chamber $C_{2}$ for $\Delta z_{2}-5 \mathrm{~mm}$ : (a) no cross talk correction and (b) with cross talk correction.

wires and the opposite cathode plane. It is seen that the Gatti calculation agrees rather well with the data. The tails of the calculated distribution are however slightly more important. One reason could be that the calcula- tion of curve $A$ was performed using a wire radius $R=15 \mu \mathrm{m}$, whereas the actual value was $10 \mu \mathrm{m}$.

Next, we displaced the central chamber by half a strip width, $\Delta z_{z}=2.5 \mathrm{~mm}$. In this position, we expect 


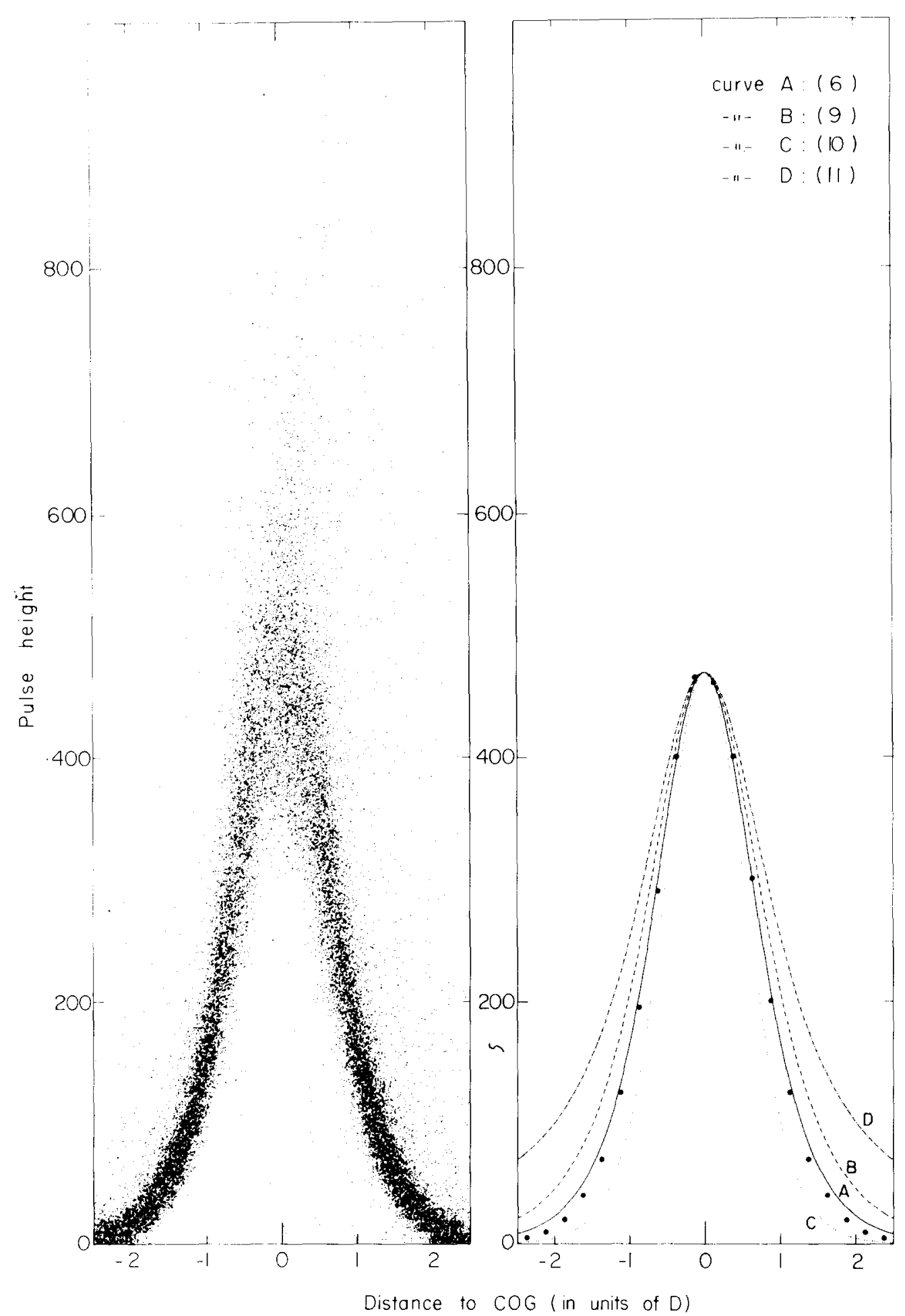

Fig. 7. (a) Measured induced charge distribution; (b) comparison of measured induced charge distribution with four model calculations. The model assumptions are described in the text.

the effect of the "internal" errors to be maximal (sect. 2). Using relation (2), we calculated the centre of the induced charges for three values of the bias parameter $b$ : $0.009,0.015,0.025$. The resulting $R_{z}$ distributions are shown in fig. $8 \mathrm{a}-\mathrm{c}$ as a function of the measured $z_{2}$ coordinate. They qualitatively agree with what we expect from our calculations. There are no sizeable systematic errors for a bias value of 0.015 . The fwhm of the 

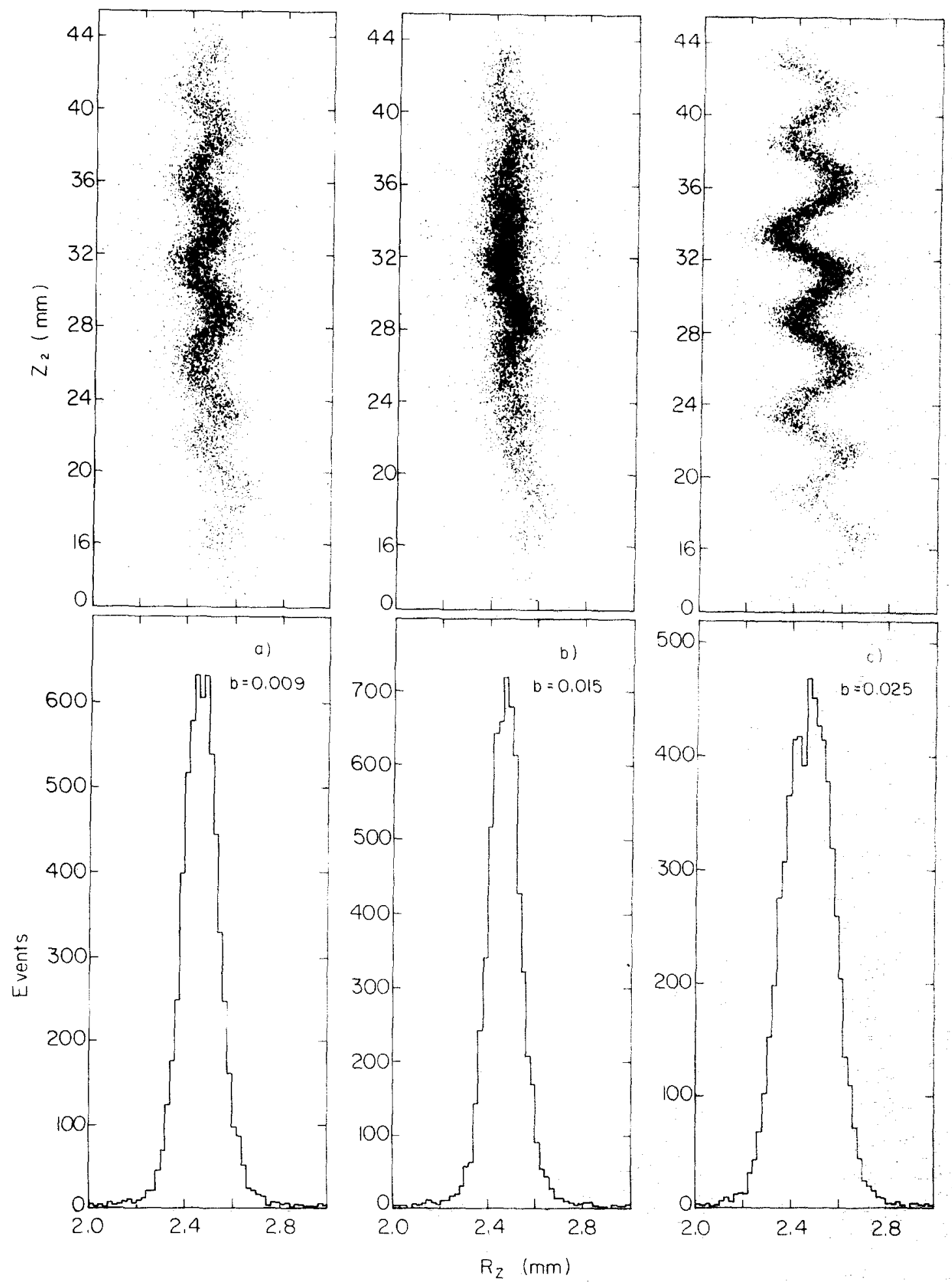

Fig. 8. Residual distribution vs the measured coordinate in chamber $C_{2}$ for $\Delta z_{2}=2.5 \mathrm{~mm}$ : (a) $b=0.009$, (b) $b=0.015$ and (c) $b=0.025$.

projected $R_{z}$ distribution is $180 \mu \mathrm{m}$, corresponding to an r.m.s. error for each plane of $62 \mu \mathrm{m}$. However, for the two other bias values, one clearly sees a periodicity in the systematic errors. We summarize in table 1 the maximum values of the systematic error calculated according to ref. 6 and experimentally found at different bias values.

However, we have seen that a small change in the 
pulse shape, induced for example by varying the parameter $s / D$ in the Gatti calculation, influences the value of the maximum systematic error. For this reason, we repeated the calculation by using our measured induced charge distribution as given in fig. 7a. The results are also given in table 1 (second line). Although a significant variation of the systematic errors is observed at the lower and higher bias values, they remain small for the two pulse shapes used in the calculation at $b=0.015$. It thus seems possible to find in our geometry a particular bias value which minimizes the maximum value of the systematic error. However, it seems advisable that the choice should be made rather by the experimental procedure, as just described, than by using the direct calculation from the Gatti pulse shape. In fact, in the latter case, one does not take into account the experimental error on the small amplitude measurements (signal/noise capability) which are of prime importance in the case where $b$ is small. This might explain the better agreement observed in table 1 at high $b$ values than at the small ones.

\subsubsection{Checks of the absolute scale}

For this purpose, we repeated the measurements of sect. 3.2.2 at different values of $\Delta z_{2}$. At each position, the residual distribution $R_{z}$ had a fwhm corresponding to an r.m.s. $\sigma$ per plane of $62 \mu \mathrm{m}$. The sizes of the reconstructed displacements are shown in fig. 9; the error on these quantities being determined only by the error on the mechanical displacement $( \pm 10 \mu \mathrm{m})$.

Two more independent checks have been made:

a) In fig. 10 we show the distribution of the difference between the coordinate measurement by a drift chamber of the UA4 experiment $[1,2]$ and the three centroid chambers. The fwhm of this distribution (160 $\mu \mathrm{m}$ ) after deconvolution of the error contribution of the drift chamber is consistent with an r.m.s. error per centroid chamber of $65 \mu \mathrm{m}$.

b) In order to intercalibrate in the NA19 experiment (fig. 11a) the positioning of the emulsion stack with respect to the beam telescope, the centroid predictions of a beam particle impact were compared to the measurements * of their corresponding interactions (star) in a thin emulsion plate $(600 \mu \mathrm{m})$. This one was placed on

Table 1

Maximum value of the systematic error $(\mu \mathrm{m})$.

\begin{tabular}{lcrl}
\hline$b$ & 0.009 & 0.015 & 0.025 \\
\hline $\begin{array}{c}\text { Calculated } \\
\quad \text { Gatti [6]) }\end{array}$ & 32 & 16 & 30 \\
Calculated & & & \\
$\quad($ fit to data) & 4 & 8 & 54 \\
Measured & 20 & $<10$ & 45 \\
\hline
\end{tabular}

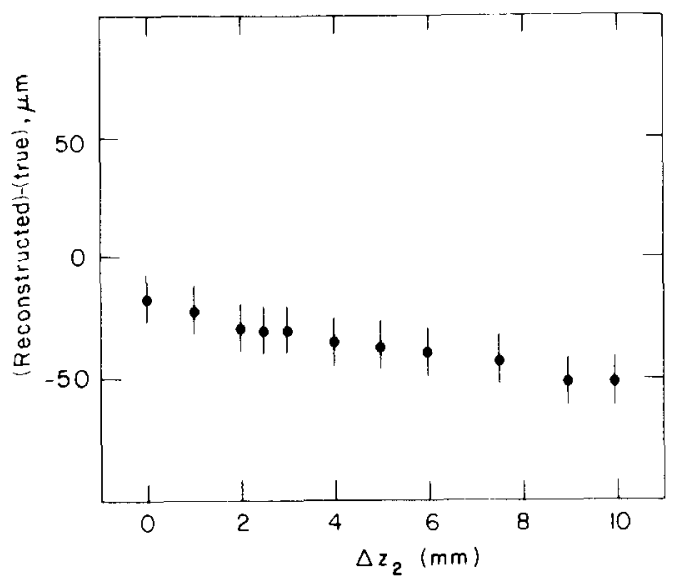

Fig. 9. Reconstructed coordinate vs displacement.

the stack holder but perpendicular to the beam direction. In addition, the density of irradiation was kept low enough to get a non-ambiguous star identification. Fig. $1 \mathrm{lb}$ shows the distribution of the difference between the chamber predictions and the star measurements. Its fwhm $(\sim 150 \mu \mathrm{m})$ is compatible with a $\sigma$ per plane of 60 $\mu \mathrm{m}$ and the geometrical propagation of this value through the sep-up. The shift of the centre of the distribution was then attributed to a misalignment in the system.

\section{Description of the centroid chamber}

\subsection{Construction of the chambers}

They are of a foam-sandwich type: the inner face of one of the cathode plates is made of a $0.1 \mathrm{~mm}$ thick fiber glass reinforced epoxy foil with metallized strips, using a conventional printed circuit technique. The other cathode is fully metallized. The strips and wire positioning is referred to external edges of the chamber and achieved with an accuracy of $20 \mu \mathrm{m}$, mainly due to the small size of the chamber: $180 \times 120 \mathrm{~mm}^{2}$ of sensitive area. The electrode material represents $\sim 1 \%$ of a radiation length.

The sense wires, $20 \mu \mathrm{m}$ in diameter, are spaced by $3 \mathrm{~mm}$, the anode-cathode distance is $5 \mathrm{~mm}( \pm 50 \mu \mathrm{m})$. The cathode strips are $4 \mathrm{~mm}$ wide, spaced by $1 \mathrm{~mm}$. To ensure a homogeneous field configuration, the last two strips and three wires close to the chamber edges are not read out. The sense wire plane was set at high voltage $(2.6-2.7 \mathrm{kV})$. The gas mixture was composed of argon, isobutane and methylal at a composition of $65-35-5 \%$ in volume. * Done by $G$. Romano from the Emulsion Group at Rome
University. 


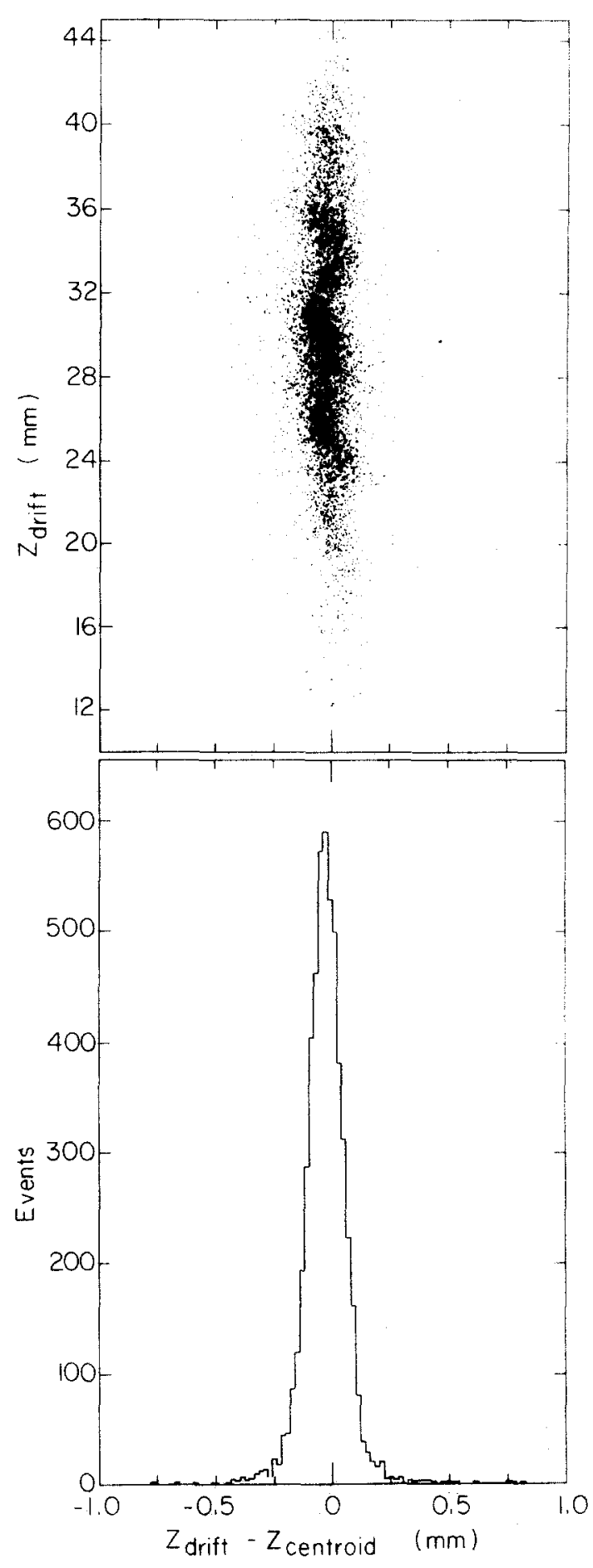

Fig. 10. Difference between the coordinate measurement by the drift chamber and the coordinate obtained from a straight line fit using the three centroid chambers, as a function of the drift coordinate. The drift chamber was placed in between two of the three equidistant $(592 \mathrm{~mm})$ centroid chambers.
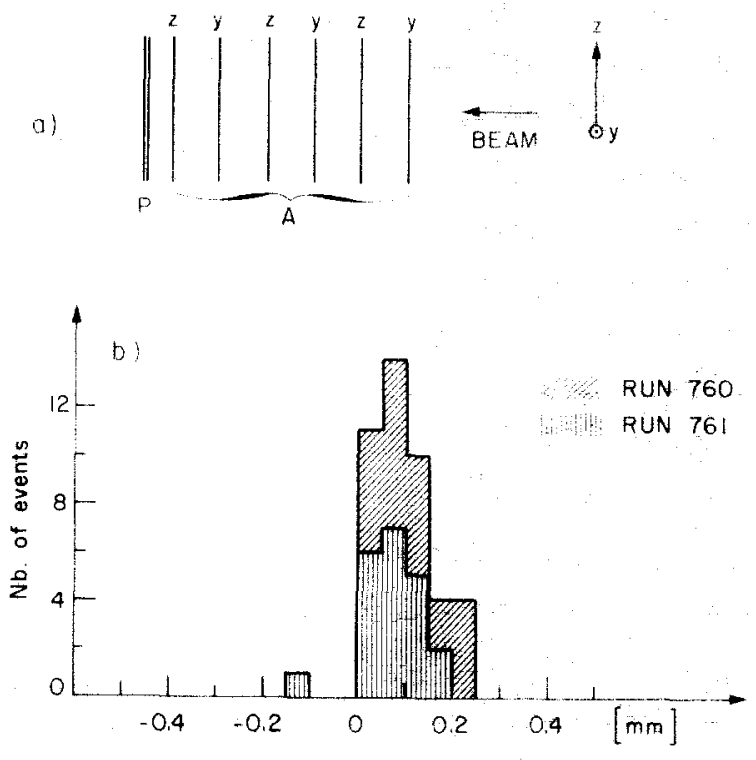

$Z$ prediction - $Z$ emulsion

Fig. 11. a) Intercalibration set-up at NA19 experiment. A Beam telescope, distance between chambers $110 \mathrm{~mm}$; the vertical pellicle $\mathbf{P}(600 \mu \mathrm{m}$ thick emulsion) is at $40 \mathrm{~mm}$ from the first chamber. b) Distribution of the difference ( $z$ coordinate) between the chamber prediction and the corresponding impact at $\mathrm{P}$.

\subsection{Electronics}

In the NA19 experiment, as well as in the reported tests, the decision to read out cathode strip pulse heights was given by an external triggering signal. These chambers were not included in any fast decision and the particle rate encountered in these experiments were moderate, at the maximum $2 \times 10^{5} / \mathrm{s} \cdot$ strip. An appropriate solution for the electronic chain is then represented in fig. 12. It consists of an integrating amplifier directly connected to the cathode strip. Its output signal is "clipped" to ground level by means of a delay line and, after a $30 \mathrm{~m}$ cable, processed into a receiving amplifier situated in the counting room. This solution showed the following characteristics:

a) A linear integration time of $200 \mathrm{~ns}$ provides a sensitivity of $2.8 \mathrm{~V} / \mathrm{pC}$ with an r.m.s. noise of $1 \mathrm{fC}$.

c) The pulse shape exhibits (fig. 13a) a "flat top" with a few percent variation in amplitude over about $100 \mathrm{~ns}$. The receiving amplifier allows an adjustment of the pulse shape and gain. To measure the pulse height, this wide pulse can be strobed in the middle of this flat top after a delay long enough to account for the time elapsed in the physics trigger decision. The charge corresponding to a 20 ns gate width is recorded by a charge sensing ADC (LRS 2249A).

The usual solution consisting of an integrator dampled by a parallel resistor was rejected for the following reasons. This choice, in addition to a larger occupation 


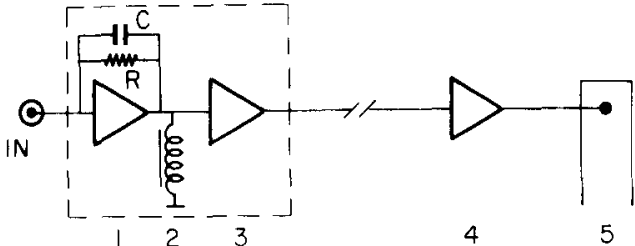

Fig, 12. Electronics for the centroid read-out: (1) integrator: CIT ALCATEL 1371; (2) delay line $200 \mathrm{~ns}, 320 \Omega$; (3) amplifier ( $\times 10$ ) line driver; (4) receiving amplifier; (5) ADC: LRS $2249 \mathrm{~A}$
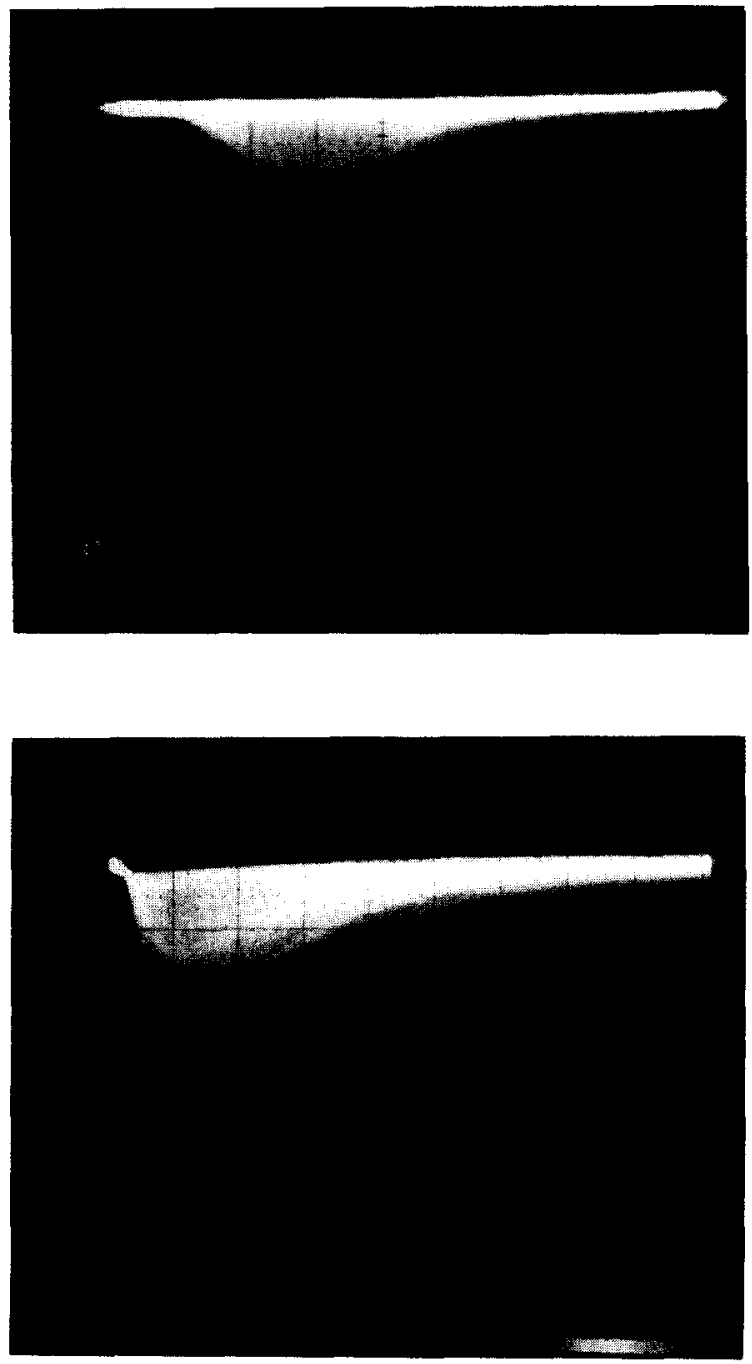

Fig. 13. Pulse shape from one strip (beam particles, $2.700 \mathrm{~V}$ ): (a) with delay line ( $100 \mathrm{~ns}, 500 \mathrm{mV})$ and (b) no delay line $(100$ ns, $100 \mathrm{mV}$ ). time $[\sim$ few $\mu s$, (fig. 13b)], is capable to amplify the signal developed at the arrival of the positive ions at the cathode: a long perturbation $(\dagger 150 \mu \mathrm{s})$, of the $\mathrm{dc}$ line is then settled which disturbs a further pulse height measurement (fig. 14). Such a scheme has been found convenient for particle rates less than $10^{4} / \mathrm{s} \cdot \mathrm{strip}$ as compared to $5 \times 10^{5}$ with the delay line solution. At this level, the ac coupling of the different elements of the chain is the most serious rate limitation.

\subsection{Remarks on the operation of the chambers}

\subsubsection{Calibration}

During the physics runs, a pedestal map of the system was frequently recorded. Its stability has been found excellent. Analog calibration was obtained by pulsing a strip capacitively coupled on the mother board to all the integrator inputs. However, from what has been said in sect. 3.2.1, it is recommended to forsee a
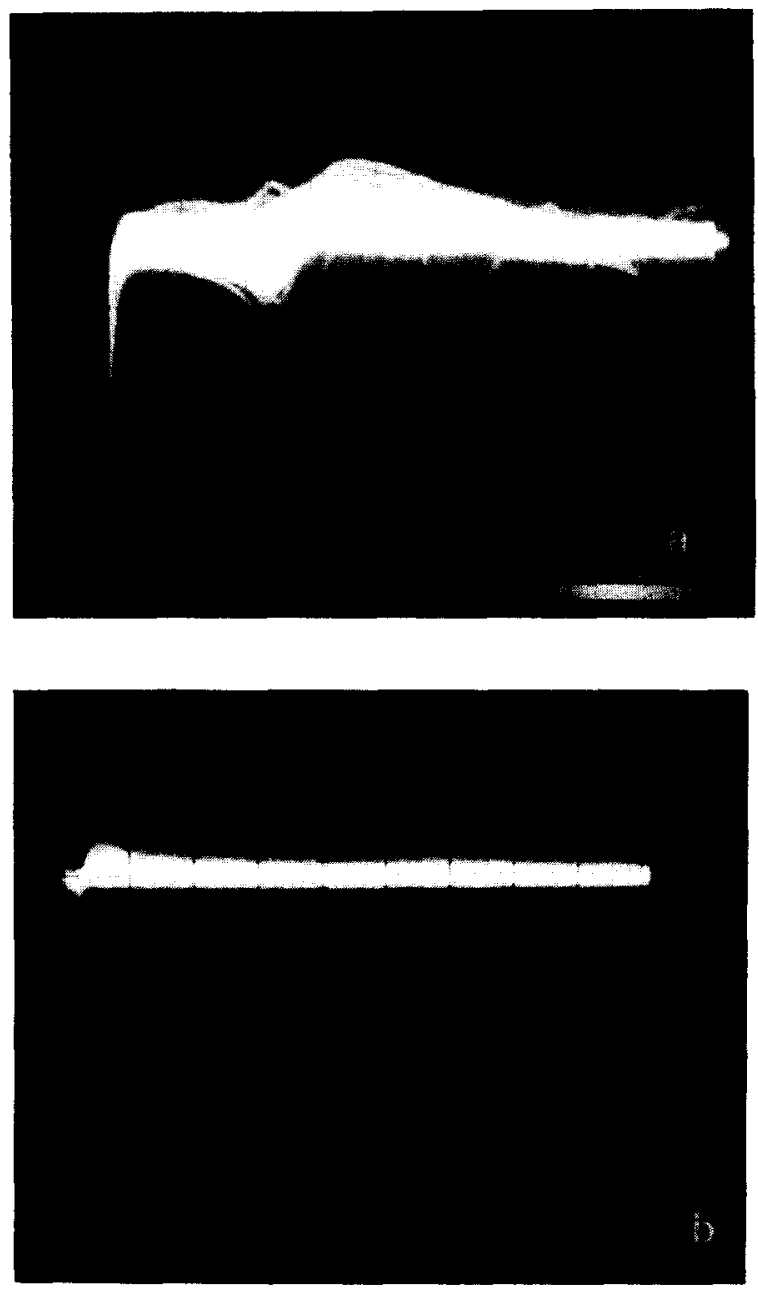

Fig. 14. Signals induced by the collection of jons at the cathode $(50 \mu \mathrm{s}, 50 \mathrm{mV}$ ): (a) no delay line and (b) with delay line. 
Table 2

Fwhm of the residual distribution for three values of the counting rate on the strips

\begin{tabular}{ll}
\hline Rate on strip $\left(\mathrm{s}^{-1}\right)$ & Fwhm $(\mu \mathrm{m})$ \\
\hline $2 \times 10^{4}$ & 130 \\
$1 \times 10^{5}$ & 150 \\
$3 \times 10^{5}$ & 200
\end{tabular}

pulsing made strip by strip for careful evaluation "in situ" of the cross talk which can be wrongly estimated by the global pulsing method.

\subsubsection{Pulse height read-out mode}

Usually, the signal from the hit wire was used to generate the ADC gate but we checked that a comparable accuracy was achieved by gating with an external signal alone. This mode of operation might allow to save a large fraction of the wire electronics.

\subsubsection{Efficiency}

A compromise between two requirements must be found for the choice of the working high voltage: a good ratio signal to noise demands for high values which conflicts the available ADC range. Any measurement in overflow (>channel 1024), among a set of hit strips degrades the accuracy of this calculated coordinate or makes it even useless, i.e. contributes to an "inefficiency' of the chamber. We adjusted the gain of the chamber in such a way as to have the most probable value of the pulse height spectrum for selected tracks passing in the middle of a strip $( \pm 1 \mathrm{~mm})$ at the $\mathrm{ADC}$ channel $400-50$. Such spectra give the most probable value of the maximum pulse height to be recorded in the system. At this condition, we have measured, in each set of three chambers, $83 \%$ of tracks with three reconstructed points and $15 \%$ with two out of three. This might be improved by using at the level of the amplifier or the ADC, non-linear elements with logarithmic or quadratic response.

\subsubsection{Rates}

The measurements with very small systematic error, i.e. $b=0.015$, have been repeated at different beam intensities. The rate effect on the fwhm of the residual distribution is given in table 2 .

\section{Conclusion}

In addition to an adequate chamber geometry, care must be taken in choosing the bias level $B$, when an algorithm such as $z=\sum_{i}\left(A_{i}-B\right) z_{i} / \Sigma_{i}\left(A_{i}-B\right)$ is used for the centre of gravity calculation. If the former choice, $2 a / D \sim 1$, minimizes stochastic errors on the localization of an avalanche along the wire, the second is shown to be related to a systematic error having a periodicity of a strip width and a maximum amplitude possibly as large as the chamber resolution. The determination of the bias level can be made by measureing the residual distribution in an array of three centroid chambers where the central one is displaced by a distance of half a strip. It is then shown that a value of $b$ (with $B=b \sum A_{i}$ ) minimizing the systematic error exists which is found equal to 0.015 in our experimental configuration. Identical results are found with a calculation where the shape of the induced charge distribution was obtained from a theoretical model (Gatti et al. [6]) or from experimental measurements. Attention must also be paid to possible contributions to the systematic error of an external source, such as electronic cross talk.

Th. Williams had to construct the chambers in a very short time and his skilfulness made their use possible at the required date. Everybody felt indebted to his excellent work and thank him.

The participation at the early phase of this study of M. Haguenauer is gratefully acknowledged together with C. Matteuzzi's contribution to the NA19 data analysis.

Finally, we thank J.C. Santiard for his efficient help in the electronic design.

\section{References}

[1] R. Battiston et al. CERN/SPSC 78-105, CERN/SPSC 79-10 and CERN/SPSC 80-59.

[2] J. Buskens et al., paper on Performance of high precision drift chambers for the UA4 Experiment (to be published).

[3] D. Allasia et al., CERN/SPSC (P 133).

[4] G. Charpak et al., Nucl. Instr. and Meth. 167 (1979) 455; G. Charpak et al., Nucl. Instr. and Meth. 148 (1978) 471.

[5] A. Breskin et al., Nucl Instr and Meth. 143 (1977) 29.

[6] E. Gatti et al, Nuct. Instr. and Meth. 163 (1979) 83.

[7] Yu Bushnin et al., Nucl. Instr. and Meth. 106 (1973) 493.

[8] V. Radeka and R.A. Boie, Nucl. Instr. and Meth. 178 (1980) 543 .

[9] I. Endo et al, HUPD-8018 preprint, Department of Physics, Hiroshima University, Hiroshima 730 . Japan (August 1980).

[10] D.E. Lee, S.E. Sobottka and H.A. Thiessen, Nucl. Instr. and Meth. 104 (1972) 179.

[11] G. Fischer and J. Plch, Nucl. Instr and Meth 100 (1972) 515. 